\title{
Mitochondrial Self-restoration as an Index to the Capacity of Avocado Fruit to Sustain Atmospheric Stress at Two Climacteric States
}

\author{
Takaya Moriguchi ${ }^{1}$ and Roger J. Romani ${ }^{2}$ \\ Department of Pomology, University of California, Davis, CA 95616 \\ Additional index words. Persea americana, mitochondria, homeostasis, stress atmospheres, mitochondrial self-restoration
}

\begin{abstract}
A strong association is implicit between mitochondrial function and the energy demands of cells responding to stress. Yet, the dynamics of this organelle-cellular dependency have been difficult to resolve. This study examines a new diagnostic parameter namely, mitochondrial maintenance and self-restoration as exhibited by the course of respiratory functions (states 3 and 4 respiratory rates, respiratory control) of mitochoudria extracted during and after exposure of intact 'Hass' avocado (Persea americana) fruit to different stress atmospheres: anoxia (100\% $\left.\mathbf{N}_{2}\right)$ or high $(25 \%$ and $75 \%)$ $\mathrm{CO}_{2}$ for varying durations. Comparisons are made with direct exposure of the mitochondria themselves to similar atmospheres. In general, exposure of the fruit to $\mathrm{CO}_{2}$ rich atmospheres enhanced the capacity of their mitochondria to restore energy-linked functions whereas anoxia caused irreparable damage. The physiological (climacteric) state of the fruit also affected the stress capacity of the mitochondria contained therein, anaerobiosis being more harmful to mitochondria in riper fruit. In contrast to their effects in vivo, in vitro anoxia appeared to sustain mitochondrial energylinked functions, whereas high $\mathrm{CO}_{2}$ was clearly harmful. These and other observations are discussed in the context of mitochondrial self-restoration or homeostasis and its relevance to postharvest stress-atmosphere storage for purposes such as pathogen suppression or insect control.
\end{abstract}

Concerns for food safety and restrictions in the use of chemicals have led to there-examination of atmospheric stress, i.e., very low levels of $\mathrm{O}_{2}$ and high levels of $\mathrm{CO}_{2}$, as means of postharvest pathogen and insect control (Ke and Kader, 1992). In such strategies, injury thresholds are critically important and one must seek to understand the bases for, and maximize the differential between, stress thresholds that result in permanent damage to the target pathogen and/or insect but in reversible damage to the stored commodity. Thus, the homeostatic potential of the commodity, its capacity to recover from stress and retain food quality, becomes a key element in the beneficial postharvest application of stressful atmospheres.

Of necessity, a stress response requires added cellular energy, which, in turn, calls for active and efficient mitochondria. Accordingly, successful recovery from stress becomes problematic if the stress conditions affect the mitochondria and compromise their ability to produce the essential cellular energy. Thus, the poststress status of mitochortdria and particularly their own homeostasis or ability to self-restore energy-linked functions may be key to defining the limits of a beneficial postharvest stress regime.

Among the earliest experiments with plant mitochondria was the germinal study, by Millered et al. (1953) with avocado mitochondria, which led to the hypothesis that uncoupling of oxidativephosphorylation and accompanying loss of energy is causal to the respiratory climacteric. That enticing interpretation was cast in doubt by later findings as was a subsequent work and hypothesis (Solomos and Laties, 1974) attributing the climacteric to the intervention of cyanide-resistant respiration and consequent diminution of cellular energy. These and many other well-executed and informative studies, e.g., the recent works by Inaba and

Received for publication 29 Aug. 1994. Accepted for publication 30 Jan. 1995. The cost of publishing this paper was defrayed in part by the payment of page charges. Under postal regulations, this paper therefore must be hereby marked advertisement solely to indicate this fact.

'Work done while a visiting scientist at UC Davis. Permanent address Fruit Research Station, Okitsu Branch, Shimizu, Shizuoka 424-02, Japan.

${ }^{2}$ To whom reprint requests should be addressed.

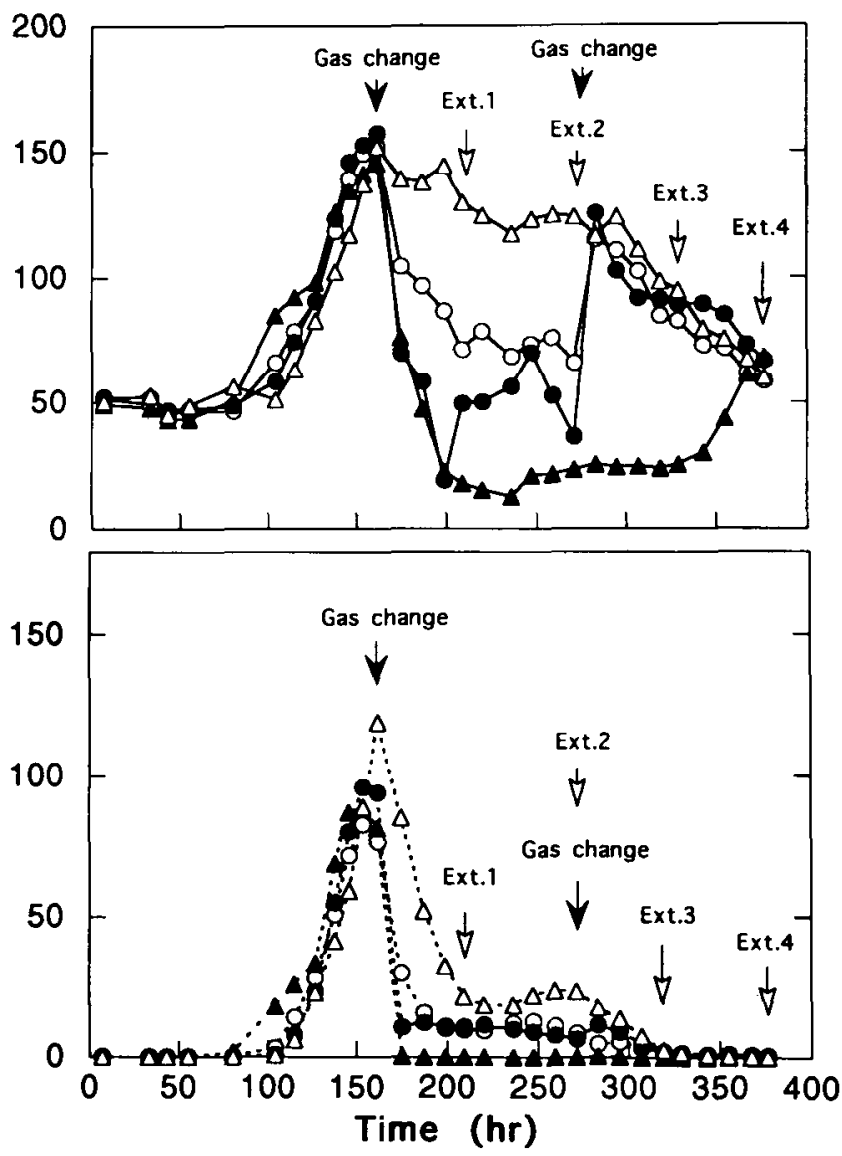

Fig. 1. Respiration (upper graph) and ethylene production (lower graph) by avocados subjected to stress atmospheres at or near the climacteric peak (first closed arrow) and returned to air $105 \mathrm{~h}$ later (second closed arrow). Air (A), 25\% $\mathrm{CO}_{2}(\mathrm{O}), 75 \% \mathrm{CO}_{2}(\bullet), \mathrm{N}_{\mathrm{z}}(\boldsymbol{\Delta})$. Mitochondria were extracted from representative fruit at four progressive stages (open arrows) 43 and $105 \mathrm{~h}$ after exposure to stress atmospheres and 58 and $105 \mathrm{~h}$ after the return to air. 
Cachin (1989) and Rahman et al. (1993), while informative have also demonstrated the difficult task of relating the metabolism of freshly isolated mitochondria to the status of the organelles in the cell.

The present study takes a different approach, one that involves repeated testing of the isolated mitochondria over time to examine whether prior in situ stress has affected their self-restorative capacity. This approach assumes that isolated mitochondria have a degree of quasi-autonomy that could include a degree of selfrestoration. Quasi-autonomy has been convincingly argued for by Margulis (1970), and mitochondrial self-restoration has recently been demonstrated by Huang and Romani (1991).

Drawing from these precepts, we sought to examine whether and to what extent exposure to stress atmospheres, either of the intact fruit or the isolated organelle, affects the capacity of mitochondria to retain or restore energy-linked functions. A preliminary report of this work has appeared (Moriguchi and Romani, 1994).

\section{Materials and Methods}

Freshly harvested preclimacteric 'Hass' avocados were obtained from the University of California's South Coast Field Station, Santa Ana, California. After selection for uniformity the fruit were placed at $25 \mathrm{C}$ in enclosed chambers and flushed with an appropriate flow ( $\approx 50 \mathrm{ml} / \mathrm{min}$ per fruit) of air or the designated stress atmosphere. A gas chromatography equipped with an alumina column and flame ionization detector was used to monitor ethylene production. Another gas chromatography equipped with appropriate columns and thermal conductivity detector was used to monitor respiration, i.e., $\mathrm{CO}_{2}$ production under air or $\mathrm{N}_{2}$ atmospheres and $\mathrm{O}_{2}$ uptake under $25 \%$ or $75 \% \mathrm{CO}_{2}$ atmospheres. All respiration data are presented as $\mathrm{CO}_{2}$ production assuming a respiratory quotient of 1 .

The $\mathrm{CO}_{2}$ atmospheres were achieved by adding $\mathrm{CO}_{2}$ to air so that the $25 \%$ and $75 \%$ atmospheres contained roughly $15 \%$ and $5 \%$ $\mathrm{O}_{2}$, respectively. Although it has been shown that even modest lowering of $\mathrm{O}_{2}$ levels can affect such important metabolic parameters as energy-charge and cytochrome $\mathrm{C}$ redox state in cultured microorganisms (Wilson et al., 1979), it is generally held that fruit metabolism, especially over the short exposures used in this study, is not significantly affected by hypoxia unless $\mathrm{O}_{2}$ levels are reduced to $1 \%$ or less (Ke and Kader, 1992). Accordingly, in presenting our data it was assumed that the stress effects of $25 \%$ or $75 \% \mathrm{CO}_{2}$ levels are due principally if not exclusively to the elevated $\mathrm{CO}_{2}$.

Mitochondria were extracted from the mesocarp of a minimum of four avocados at the designated time during and after exposure to atmospheric stress. Avocados at or near the climacteric peak were used to obtain the large amounts of mitochondria required for

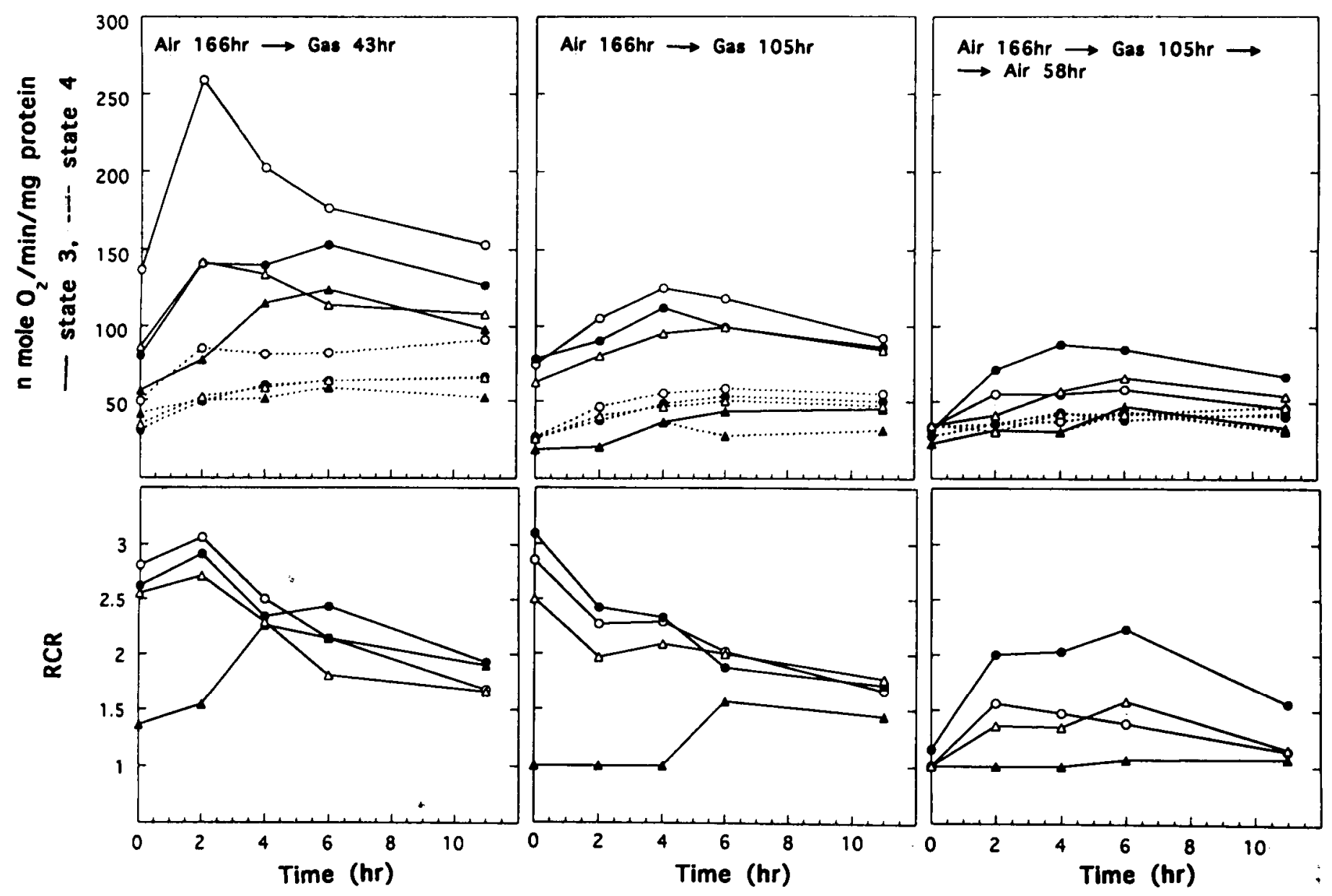

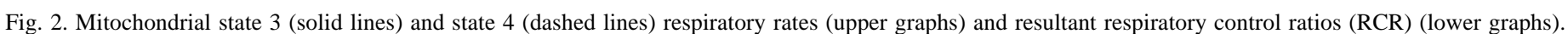

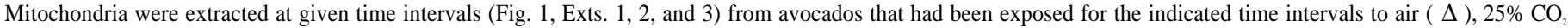
$(\bigcirc), 75 \% \mathrm{CO},(\boldsymbol{O})$, or $\mathrm{N}_{2}(\boldsymbol{\Delta})$. After extraction, the mitochondria were incubated and their respiratory functions were assayed under aerobic conditions. 


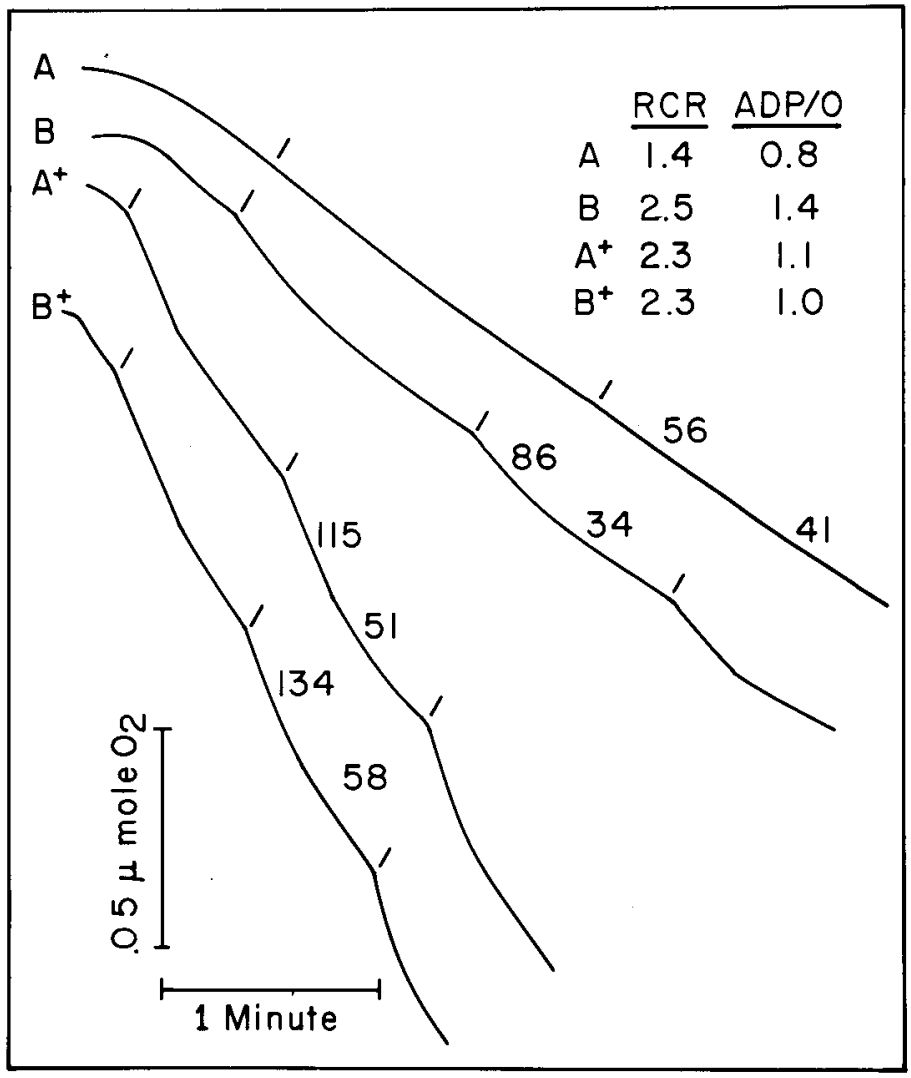

Fig. 3. Polarographic traces depicting $\mathrm{O}_{2}$ consumption by avocado mitochondria. Mitochondria were assayed immediately after extraction from avocados held under $\mathrm{N}_{2}$ for $43 \mathrm{~h}$ (A) or from air-treated control fruit (B). The same mitochondria assayed again after $4 \mathrm{~h}$ of incubation under aerobic conditions $\left(\mathrm{A}^{+}, \mathrm{B}^{+}\right)$. Small slant lines denote the addition of $0.05 \mu \mathrm{mol}$ of ADP. Numbers refer to respiration rate (nmoles $\mathrm{O}_{2} / \mathrm{min}$ per mg protein) in the presence of ADP (state 3 ) and after the added ADP has been consumed (state 4).

direct exposure of the organelles to stress atmospheres, in vitro. In all instances, mitochondria were extracted from fruit using medium and procedures described in detail elsewhere (Romani et al., 1974). Firm (preclimacteric) tissue was grated while submersed in isolation medium, softer (climacteric) tissue was pressed through a screen submersed in medium. The mitochondria were once washed, i.e., underwent one cycle of resuspension and centrifugation.

The freshly prepared mitochondria were placed in reaction medium $(0.25 \mathrm{~m}$ sucrose, $0.1 \% \mathrm{BSA}, 50 \mathrm{~mm}$ phosphate [pH 7.2], $10 \mathrm{~mm} \alpha$ - ketoglutarate, $1 \mathrm{~mm} \mathrm{MgCl}, 10 \mu \mathrm{M} \mathrm{CoA,} 100 \mu \mathrm{M} \mathrm{TPP,} 100$ $\mu \mathrm{M} \mathrm{NAD}$, and $100 \mu \mathrm{g} \cdot \mathrm{ml}^{-1}$ chloramphenicol) at a concentration of 0.2 to $0.4 \mathrm{mg}$ mitochondrial protein $/ \mathrm{ml}$. The suspension was placed in small $(20-$ or $40-\mathrm{ml})$ beakers on a rotary shaker at $25 \mathrm{C}$ with the speed set just sufficient for gentle swirling. To monitor mitochondrial functions, $1 \mathrm{ml}$ of the suspended mitochondria was place in a plexiglass chamber equipped with a Yellow Springs Instruments oxygen electrode. When too low to permit an informative assay, the $\mathrm{O}_{2}$ level in the 1-ml aliquot was raised with gentle bubbling of air for 30-60 sec before the chamber was closed. Oxygen consumption was measured before and after the addition stoichiometric amounts of ADP. Respiratory control (RC) and $\mathrm{ADP} / \mathrm{O}$ were obtained from the recorder traces. A quantitative estimate of RC, the respiratory control ratio (RCR) equals the respiratory rate in state 3 divided by that in state 4 . These standard procedures are described in detail in Ozelkok and Romani (1975).

For in vitro exposure to stress atmospheres, mitochondria were suspended in reaction mixture in concentrations similar to those
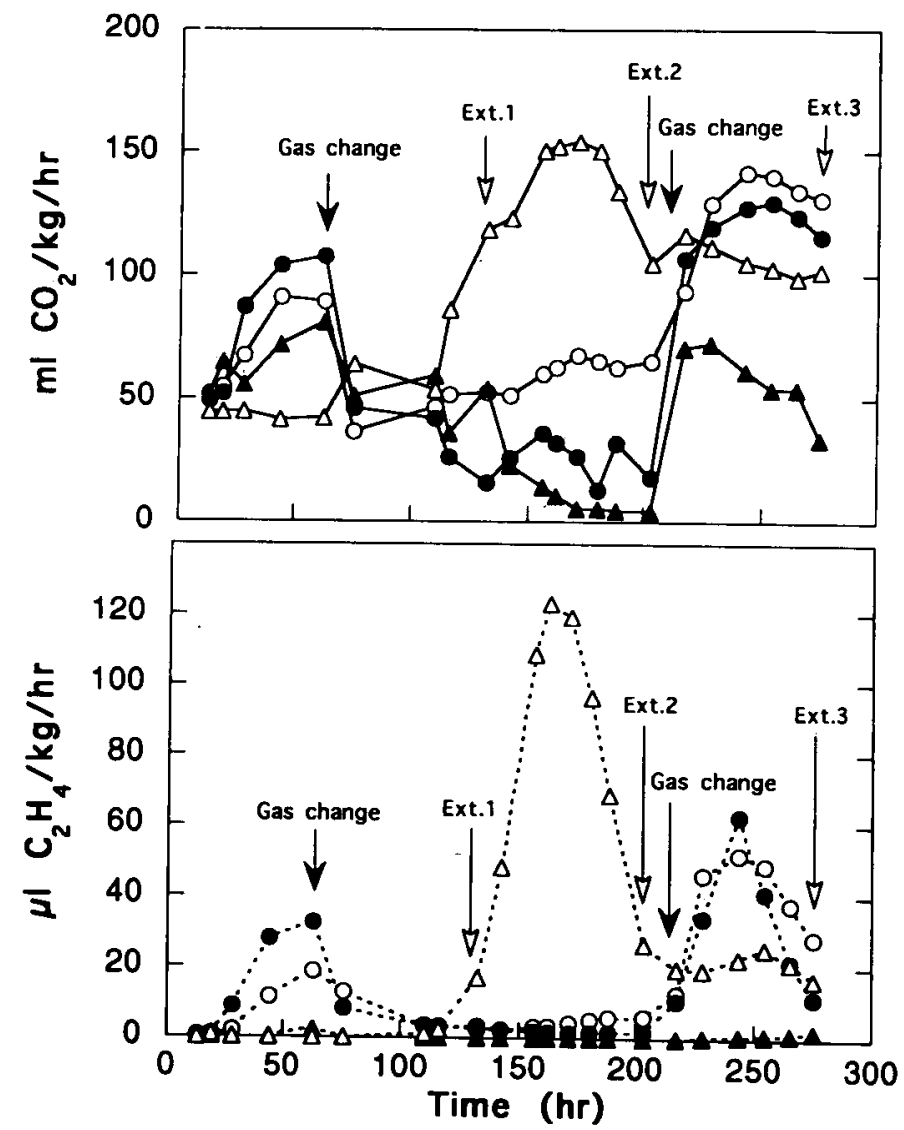

Fig. 4. Respiration (upper graph) and ethylene production (lower graph) by avocados subjected to stress atmosphere early in their climacteric rise (first closed arrow) and returned to air $147 \mathrm{~h}$ later (second closed arrow). Air $(\Delta) 25 \% \mathrm{CO}_{2}(\mathrm{O})$, $75 \% \mathrm{CO}_{2}(\bullet), \mathrm{N}_{2}(\boldsymbol{\Delta})$. Mitochondria were extracted from representative fruits at 3 progressive stages (open arrows), 69 and $140 \mathrm{~h}$ after exposure to stress atmospheres and $65 \mathrm{~h}$ after subsequent return to air.

used for monitoring $\mathrm{O}_{2}$ consumption. A fixed volume ( 10 or $20 \mathrm{ml}$ ) of the mitochondrial suspension was placed in each of four 50-ml Erlenmeyer flasks held at $25 \mathrm{C}$ on a rotary shaker adjusted to achieve gentle swirling. Each flask was closed with a serum cap equipped with inflow and outflow lines to the headspace. Air or the designated stress atmosphere $\left(\mathrm{N}_{2}, 25 \% \mathrm{CO}_{2}\right.$, or $\left.75 \% \mathrm{CO}_{2}\right)$ was flushed through the headspace at $20 \mathrm{ml} \cdot \mathrm{min}^{-1}$. To test for energylinked functions at the indicated time intervals a one $\mathrm{ml}$ aliquot of mitochondrial suspension was taken slowly using a 2-ml syringe and a 16-gauge needle so as not to disturb the stress atmosphere or physically stress the mitochondria.

A modified Lowry procedure (Miller, 1979) was used for protein determinations using serum albumin as the standard. Several exploratory and three comprehensive experiments were run with intact fruit; data from two (Figs. 1, 2, 4, and 5) are presented. Three complete replicates of the in vitro studies were conducted with comparable results; data from one are shown (Fig. 6).

\section{Results and Discussion}

Atmospheric stress in vivo. Seen in Fig. 1 are the respiratory and ethylene production rates of avocados held at $25 \mathrm{C}$ in air and then subjected, at or near their climacteric peak, to the indicated atmosphere for a specified length of time before being returned to air. The atmospheric conditions, particularly anoxia $\left(\mathrm{N}_{2}\right)$, were chosen more to exacerbate cellular stress than to simulate posthar- 
vest practices, which are likely to be less severe. Timing of the stress. at or near the climacteric peak. was chosen to lessen the impact of rapid physiological transitions that accompany the climacteric rise. The observed respiration rates reflect the anticipated effects of atmospheric stress. Suppressed roughly in proportion to imposed $\mathrm{CO}_{2}$ levels, respiration returns to near normal after return to air. However, the very low level of $\mathrm{CO}_{2}$ production during anoxia, likely to have been largely fermentative, remains suppressed after the return to air at $271 \mathrm{~h}$. The final rise in $\mathrm{O}_{2}$ consumption beginning at $360 \mathrm{~h}$ is attributable to microbial infection.

Under $25 \%$ and $75 \% \quad \mathrm{CO}_{2}$, a low level $\left(4-10 \mu \mathrm{l} \cdot \mathrm{kg}^{-1} \cdot \mathrm{h}^{-1}\right)$ of ethylene production was maintained. There was no measurable ethylene production under anoxia nor after return of the anoxic fruit to air. The $25 \% \mathrm{CO}_{2}$ treatment resulted in no off-color or offflavor but a retardation of ripening. Mild discoloration and possibly some off-flavor were discernable after the $75 \% \mathrm{CO}$, treatment.

The effects of these modified atmospheres on the-restorative capacity of mitochondria derived from the stressed tissues are summarized in Fig. 2. Shown are the state 3 (in the presence of ADP) and state 4 (after depletion of the added ADP) respiratory rates and the resultant level of RC of mitochondria immediately after their extraction from the fruit tissues and at several times for $10+\mathrm{h}$ thereafter, during which the isolated mitochondria were incubated under air.

Each of the data points in Fig. 2 was taken from individual polarographic traces of $\mathrm{O}_{2}$ consumption such as those shown in Fig. 3, where it is seen that, immediately after extraction from fruit exposed to $\mathrm{N}_{2}$ for $43 \mathrm{~h}$ (trace $\mathrm{A}$ ), the mitochondria had low rates of $\mathrm{O}_{2}$ uptake and very low $\mathrm{RC}(\mathrm{RCR}=1.4)$ but were able to double their state 3 respiratory rate and restore energy-linked function $(\mathrm{RCR}=2.3)$ after $4 \mathrm{~h}$ at $25 \mathrm{C}$ (trace $\mathrm{A}+)$. Mitochondria from airtreated fruit had good $\mathrm{RC}$ and $\mathrm{ADP} / \mathrm{O}$ when freshly isolated (trace B) and, although their $\mathrm{O}_{2}$ uptake increased, their $\mathrm{RC}$ declined slightly after $4 \mathrm{~h}$ (trace $\mathrm{B}+$ ). $\mathrm{RC}$ is an indirect measure of the extent to which mitochondria can generate a protonmotive force that suppresses respiration (state 4) and is a good index of mitochondrial integrity. With the addition of ADP, protons flow back into the mitochondrial matrix via the ATPase complex, ATP is formed for cellular use, the protonmotive force is dissipated, and respiratory rate increases (state 3 ). Injured mitochondria generally have a proton leaky inner membrane, do not generate a protonmotive force, and therefore generate little if any ATP for their own or cellular use. Such mitochondria would be incapable of supporting cell maintenance or recovery from transient stress conditions. Our aim was to examine to what extent such mitochondrial dysfunction is brought about by atmospheric stress, whether the function can be restored by the mitochondria, i.e., self-restoration, and to what extent, if any, the dynamics of mitochondrial self-restoration relate to the response of the intact fruit.

As shown in Fig. 2, there was an overall decline in states 3 and 4 rates of $\mathrm{O}_{2}$ consumption and in $\mathrm{RC}$, as mitochondria are extracted

Ext. 3
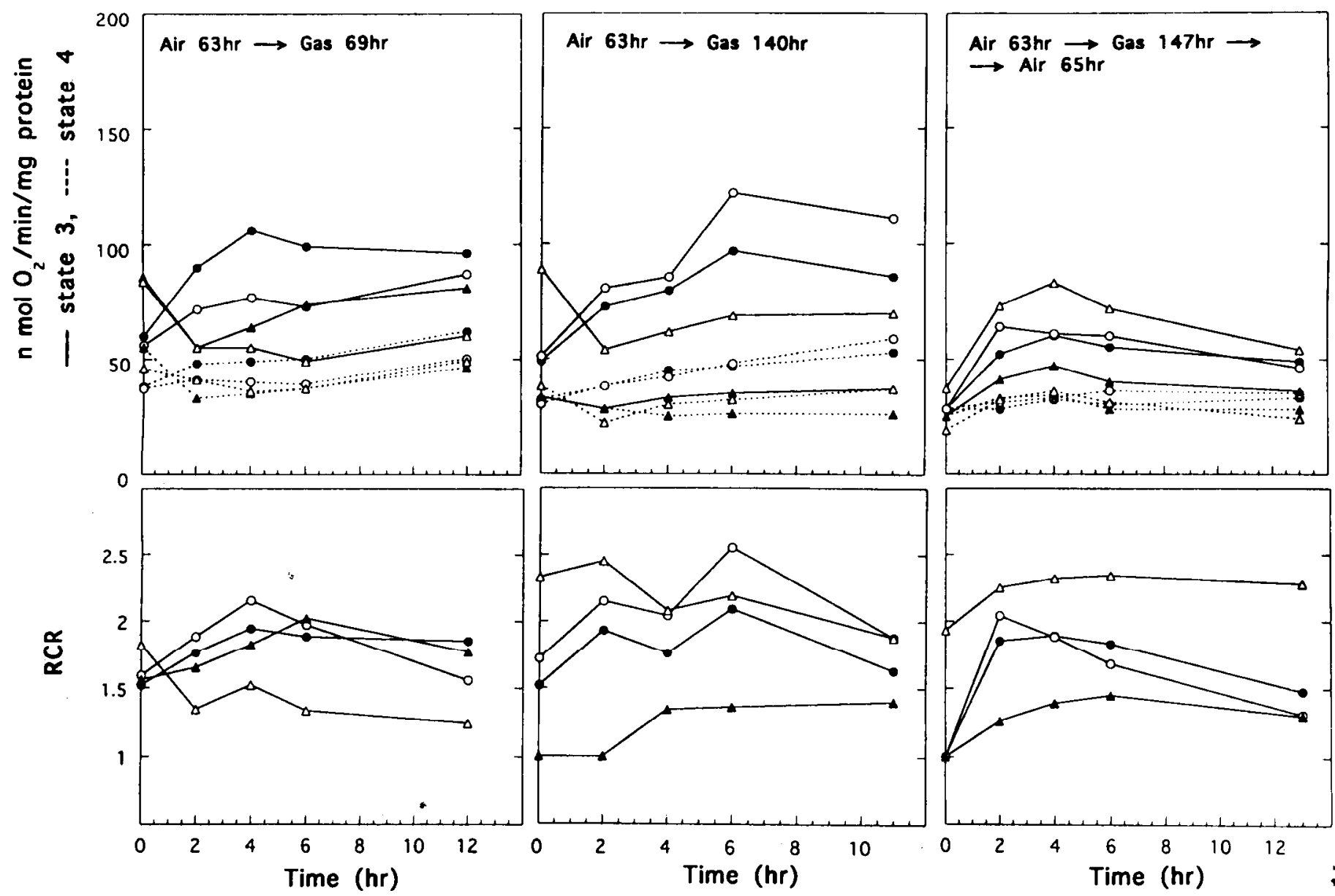

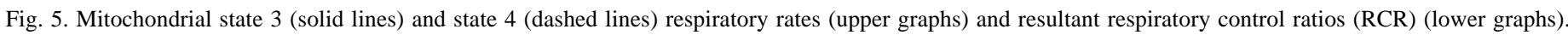

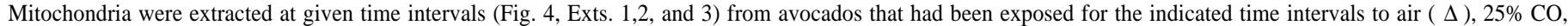
$(\bigcirc), 75 \% \mathrm{CO}_{2}(\boldsymbol{O})$, or $\mathrm{N}_{2}(\boldsymbol{\Delta})$. After extraction the mitochondria were incubated and their respiratory functions assayed under aerobic conditions. 


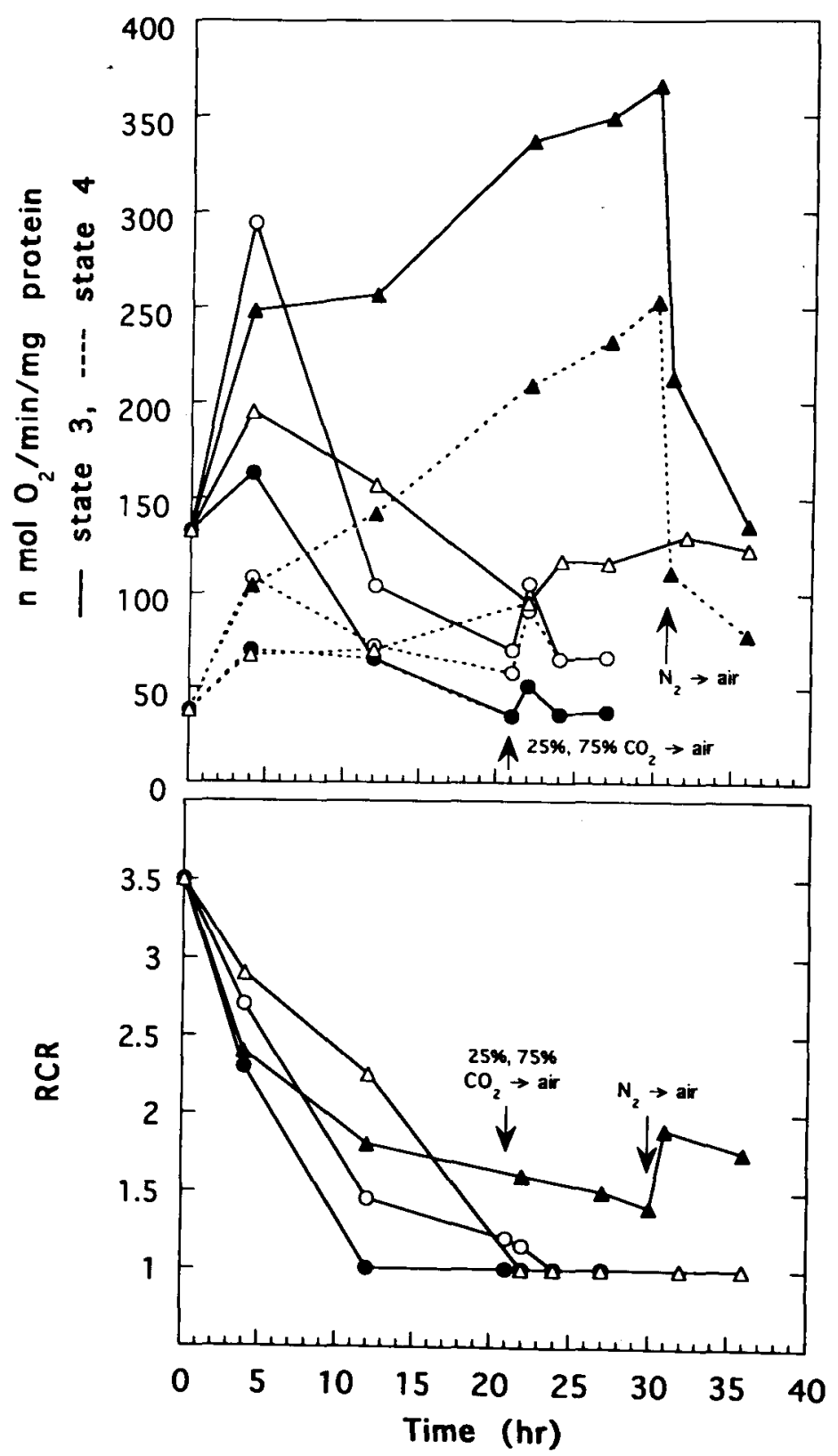

Fig. 6. Respiratory rates and respiratory control ratios (RCR) of mitochondria extracted from avocados at or near their climacteric peak. The mitochondria were incubated in reaction medium in equilibrium with air $(\mathrm{A}), 25 \% \mathrm{CO}_{2}(\mathrm{O}), 75 \% \mathrm{CO}_{2}$ ( ) , or $\mathrm{N}_{2}(\boldsymbol{\Delta})$. After $21 \mathrm{~h}$ (first arrow), both $\mathrm{CO}_{2}$ atmospheres were replaced by air; after $30 \mathrm{~h}$ (second arrow), $\mathrm{N}_{2}$ was replaced by air. Each datum point is derived from measurement of $\mathrm{O}_{2}$ consumption made immediately after an aliquot of the incubating mitochondria was gently perfused with air.

from progressively older, postclimacteric avocados. By the fourth extraction, $376 \mathrm{~h}$ after the start of the experiment (data not shown), states 3 and 4 rates were even further depressed and RC was absent $(\mathrm{RCR}=1)$, with no restoration of either function during the postextraction incubation of the mitochondria.

In spite of this general decline as fruit overripened, mitochondria from each of the first three extractions exhibit some capacity to restore respiratory activity during the several hours of incubation in vitro. Mitochondria taken from fruit treated with $\mathrm{CO}_{2}$ maintained RC levels similar to the controls. By the third extraction, only the mitochondria extracted from $75 \% \mathrm{CO}_{2}$-treated fruit showed an appreciable capacity to restore RC. One could question whether the sparing effect of high $\mathrm{CO}_{2}$ atmospheres is simply the consequence of delayed senescence, but that seems unlikely in view of the fact that the post-treatment respiratory pattern of the intact fruit (Fig. 1) quantitatively and temporally duplicates that of the control fruit. It appears, therefore, that exposure of climacteric avocados to high $\mathrm{CO}_{2}$ levels for up to $105 \mathrm{~h}$ (4+ days) does not compromise the ability of their mitochondria to serve as sources of essential cellular energy. The implication is that the cells will be supplied with energy to reverse the damaging effects of $\mathrm{CO}_{2}$ stress and return to a near-normal physiological state-as would appear to have been the case in terms of overall respiratory activity (Fig. 1).

In contrast to the protective effects of $\mathrm{CO}_{2}$, mitochondria taken from fruit subjected to $105 \mathrm{~h}$ of anoxia (Fig. 2, Ext. 2) had no RC for the first $4 \mathrm{~h}$ and only a faint restoration of this function by $6 \mathrm{~h}$ of in vitro aerobiosis. This debilitating effect of anoxia on the ability of mitochondria to restore energy-linked functions would explain why initochondria extracted $58 \mathrm{~h}$ after the anoxic fruit had been returned to air (Fig. 2, Ext. 3) had completely lost the ability to restore RC. This condition is mirrored in the behavior of the intact fruit (Fig. 1), whose respiration, except for that attributable to infection in the latter stages, remains suppressed.

Shown in Fig. 4 are the respiration and ethylene production rates of avocados exposed to stress atmospheres at or just past the onset of their climacteric rise. As one might surmise from early experiments on the response of preclimacteric avocados to modified atmospheres (Young et al., 1962), anoxia and the two $\mathrm{CO}_{2}$ atmospheres suppressed the respiratory and ethylene climacterics. Upon transfer to air $147 \mathrm{~h}$ later, the $\mathrm{CO}_{2}$-treated fruit exhibited an accelerated respiratory rise reaching near normal climacteric peak respiratory rates with an accompanying but suppressed ethylene climacteric. The $\mathrm{N}_{2}$-treated fruit exhibited a modest respiratory rise but no increase $m$ ethylene production upon transfer to air. This fact, plus obvious signs of discoloration and injury, would suggest that these $\mathrm{N}_{2}$-treated tissues were irreparably dysfunctional. The time course of energy-linked functions of mitochondria extracted at different times from these variously treated fruit are shown in Fig. 5.

Mitochondria from the $\mathrm{CO}_{2}$ treated fruit, after either the 69(Ext. 1) or 140-h (Ext. 2) exposure, showed considerable improvement in state 3 respiratory rates during their first $6 \mathrm{~h}$ of incubation in vitro. This in vitro after-affect of prior in vivo exposure to elevated $\mathrm{CO}_{2}$ mirrors, and perhaps is causal to, the rapid rise in respiration of the intact fruit upon return to air (Fig. 4). When extracted from fruit $65 \mathrm{~h}$ post- $\mathrm{CO}_{2}$ treatment (Ext. 3), mitochondria had very low respiratory rates initially and, although the rates increased during the first $2 \mathrm{~h}$ in vitro, they never reached the levels of the mitochondria from control fruit. Oxygen consumption of mitochondria from $\mathrm{N}_{2}$-treated fruit was even further suppressed, again mirroring the behavior of the intact fruit (Fig. 4).

At the first extraction ( $63 \mathrm{~h}$ air $+69 \mathrm{~h}$ stress atmosphere), the RC of control mitochondria was lower than that of the treated fruit (Fig. 5). The apparent anomaly may be explained by the fact that mitochondria with higher $\mathrm{RC}$ and better $\mathrm{RC}$ maintenance are obtained from progressively riper avocados up to the climacteric peak (Ozelkok and Romani, 1975). Accordingly, both initial RC and RC maintenance by control mitochondria improved and was highest by the third extraction. Mitochondria from fruit exposed to high $\mathrm{CO}_{2}$ for $63 \mathrm{~h}$ (Ext. 2) exhibited and retained good RC but, when extracted from fruit $65 \mathrm{~h}$ post-treatment (Ext. 3), the recovery in RC, although dramatic, was short-lived. This may imply that $\mathrm{CO}_{2}$ treatment of these preclimacteric fruit impaired their mitochondria's capacity for self-restoration. Comparison with the performance of mitochondria from $\mathrm{CO}_{2}$-treated climacteric fruit 
Table 1. The $\mathrm{pH}$ of the mitochondrial incubation medium after exposure to various gas atmospheres and at various intervals upon subsequent return to air.

\begin{tabular}{lcccc}
\hline & & \multicolumn{3}{c}{$\mathrm{pH}$ after transfer to air for } \\
\cline { 2 - 5 } & & $1 \mathrm{~h}$ & $3 \mathrm{~h}$ & $6-8 \mathrm{~h}$ \\
\hline $21 \mathrm{~h} 25 \% \mathrm{CO}_{2}$ & 6.55 & 6.90 & 7.05 & 7.25 \\
$21 \mathrm{~h} 75 \% \mathrm{CO}_{2}$ & 6.30 & 6.75 & 7.15 & 7.15 \\
$30 \mathrm{~h} \mathrm{~N}_{2}$ & 7.20 & 7.20 & --- & 7.25 \\
\hline
\end{tabular}

${ }^{\mathrm{p} H}$ of incubation medium at end of the exposure period ( $\mathrm{pH}$ of the medium before exposure $=7.2$.).

(Fig. 2) would suggest that the latter fruit are less susceptible to $\mathrm{CO}_{2}$ stress.

The injurious effects of $\mathrm{N}_{2}$ treatment are obvious by the very low or complete absence of RC in the freshly extracted mitochondria. It may be noted, however, that even after the long term (147 h) exposure to $\mathrm{N}_{2}$ and subsequent air treatment of the fruit, mitochondria (Fig. 5, Ext. 3) initially devoid of RC demonstrated some restoration of it during in vitro incubation. No such restoration was shown by mitochondria taken from riper avocados were subjected to a similar atmospheric stress (Fig. 2, Ext. 3). This difference in mitochondrial performance may explain the difference in whole-fruit respiration, i.e., no recovery after anoxic treatment of climacteric fruit (Fig. 1) as opposed to $\approx 50 \%$ recovery after similar treatment of the less mature fruit (Fig. 4).

Atmospheric stress in vitro. For comparative purposes, sound mitochondria $(\mathrm{RCR}=3.5)$ were extracted from avocados at or near their climacteric peak, resuspend in reaction medium, and exposed to air or stress atmospheres. Periodically, 1-ml aliquots of the incubating mitochondria were gently aerated and assayed for respiratory function. The results of one such typical experiment are summarized in Fig. 6. Several respiratory trends are observed.

With incubation of the extracted mitochondria in air an increase in state 3 respiratory activity during the first $4 \mathrm{~h}$ was followed by a gradual decline over the next $16 \mathrm{~h}$. B y $20 \mathrm{~h}$, the mitochondria had lost respiratory control. Mitochondria exposed to $25 \%$ or $75 \% \mathrm{CO}_{2}$ evidenced a somewhat suppressed but similar respiratory trend and a total and permanent loss of RC after $\approx 24 \mathrm{~h}$ of $25 \% \mathrm{CO}_{2}$ or 12 $\mathrm{h}$ of $75 \% \mathrm{CO}_{2}$ Acidification of the medium is anticipated upon exposure to elevated levels of $\mathrm{CO}_{2}$. As seen in Table 1, acidification did occur, but returned to near normal $\mathrm{pH}$ in the subsequent first hour in an air atmosphere. This relatively rapid readjustment, plus the fact that mitochondrial activity is usually little compromised by the observed shifts in $\mathrm{pH}$, makes it unlikely that $\mathrm{pH}$ change per se is a factor in the dysfunctions caused by $\mathrm{CO}_{2}$.

In contrast to the injurious effects of $\mathrm{CO}_{2}$, the respiratory rates of mitochondria incubated under $\mathrm{N}_{2}$ continued to increase, while measurable RC was maintained during the 30 -h exposure. Upon transfer to air after $30 \mathrm{~h}$ of $\mathrm{N}_{2}$, states 3 and 4 rates declined rapidly, but again differentially so that an actual increase in RCR was observed.

The striking increase in states 3 and 4 rates following progressive exposure to anoxia is likely the result of accumulated metabolic intermediates arising from a reductive partial TCA cycle which has been found to be operative in hypoxic pears (Nanos et al., 1994). The intermediates would be quickly consumed upon exposure to air. In any event, it is clear that the electron transport and energy conserving functions of the mitochondria are not harmed by their direct exposure to $\mathrm{N}_{2}$ for $30 \mathrm{~h}$. Accordingly, one may assume that mitochondrial injury in fruit exposed to $\mathrm{N}_{2}$ is not the direct effect of anaerobiosis but its influence via an altered and less favorable intracellular milieu.

\section{Limitations and Prospects}

The approach taken in this study has promise and limitations, including the need for many assays of mitochondrial function to follow the postisolation behavior of the organelles, e.g., the data in Fig. 2 result from 16 separate extractions of mitochondria and 80 polarographic assays. Meaningful assessment of stress effects requires that the reference mitochondria retain energy-linked functions for survival for some hours against which one can then discerns the harmful effects of imposed stress, in vivo or in vitro. Although not used in the present study, further purification of the mitochondria via a Percoll pad or self-forming Percoll gradient can extend mitochondrial survival. Repelleting and resuspension of the incubating mitochondria in fresh medium can also extend survival to several days (Romani et al., 1974). Such steps would have increased the diagnostic potential of the isolated organelles but would also have amplified the experimented protocol and required yet larger extractions, since each of these steps involves the loss of mitochondria.

A second constraint derives from what is unquestionably a complex, interdependent relationship between mitochondria and the cell [see Ricard et al. (1994) for an informative review]. Intracellular interpendency is implicit in the markedly different affects on mitochondrial function seen when the same atmospheric stress is applied to the intact fruit as opposed to mitochondria in suspension. Nonetheless, given that energy demanding repair is essential if short-term, high-stress atmospheres are to be effective postharvest regimes, it is this very cellular-mitochondrial dependency that needs to be better understood.

We have drawn attention to some to of the observed relationships between type and duration of atmospheric stress, the physiological state of the fruit, and the self-restorative capacity of mitochondria extracted from the stressed fruit. Although more experimentation and refined experimental protocols are called for, we believe the present study clearly demonstrates that repeated analyses of mitochondrial function overtime yield more different and likely true-to-life information than that obtained from a onetime assessment of the organelles immediately after extraction. This leads us to suggest that the study of mitochondrial selfrestoration can result in useful insights on the role of mitochondria in stressed cells and, in turn, on the cellular limits for stress.

\section{Literature Cited}

Huang, L.S. and R.J. Romani. 1991. Metabolically driven self-restoration of energy-linked functions by avocado mitochondria. Plant Phyisol. 95:1096-1105.

Inaba, M. and K. Chachin. 1989. High-temperature stress and mitochondrial activity of harvested mature-green tomatoes. J. Amer. Soc. Hort. Sci. 114:809-814.

Ke, D. and A.A. Kader. 1992. Potential of controlled atmospheres for postharvest insect disinfestation of fruits and vegetables. Postharvest News Info. 3:31N-37N.

Margulis, L. 1970. Origin of eucaryotic cells. Yale University Press, New Haven, Corm.

Miller, G.L. 1979. Protein determination for large number of samples. Anal. Chem. 31:964.

Millered, A., J. Bonner, and J.B. Biale. 1953. The climacteric rise in fruit respiration as controlled by phosphorylative coupling. Plant Physiol. 28:521-531.

Moriguchi, T. and R.J. Romani. 1994. Respiratory functions of avocado fruit mitochondria during and after exposure to high $\mathrm{CO}_{2}$ or anoxic atmospheres, in vivo and in vitro. XXIVth Intl. Hort. Congr. p. 272. (Abstr.)

Nanos, G. N., R.J. Romani, and A.A. Kader. 1994. Respiratory metabolism of pear fruit and cultured pear fruit cells exposed to hypoxic 
atmospheres: Associated changes in activities of key enzymes. J. Amer. Soc. Hort. Sci. 119:288-294.

Ozelkok, S.I. and R.J. Romani. 1975. Ripening and in vitro retention of respiratory control by avocado and pear mitochondria. Plant Physiol. 56:239-244.

Rahman, A. A., D.J. Huber, and J.K. Brecht. 1993. Respiratory activity and mitochondrial oxidative capacity of bell pepper fruit following storage under low-oxygen atmosphere. J. Amer. Soc. Hort. Sci. 118:470475.

Ricard, B., I. Couée, P. Raymond, P.H. Saglio, V. Saint-Ges, and A. Pradet. 1994. Plant metabolism under hypoxia and anoxia. Plant Physiol. Biochem. 32:1-10.
Romani, R.J., S.E. Tuskes and S. Ozelkok. 1974. Survival of plant mitochondria in vitro: Form and function after 4 days at 25C. Arch. Biochem. Biophys. 164:743-751.

Solomos, T. and G.G. Laties. 1974. Similarities between the action of ethylene and cyanide in initiating the climacteric and ripening of avocados. Plant Physiol. 28:279-297.

Wilson, D. F., M. Erecinka, C. Brown, and I.A. Siber. 1979. The oxygen dependence of cellular metabolism. Arch. Biochem. Biophys. 195:485493.

Young, R. E., R. J. Romani, and J. B. Biale. 1962. Carbon dioxide effects on fruit respiration. II. Response of avocados, bananas and lemons. Plant Physiol. 37:416-422. 